\title{
Ultranarrow polaritons in a semiconductor microcavity
}

\author{
Jensen, Jacob Riis; Borri, Paola; Langbein, Wolfgang; Hvam, Jørn Märcher
}

Published in:

Applied Physics Letters

Link to article, DOI:

10.1063/1.126601

Publication date:

2000

Document Version

Publisher's PDF, also known as Version of record

Link back to DTU Orbit

Citation (APA):

Jensen, J. R., Borri, P., Langbein, W., \& Hvam, J. M. (2000). Ultranarrow polaritons in a semiconductor microcavity. Applied Physics Letters, 76(22), 3262-3264. https://doi.org/10.1063/1.126601

\section{General rights}

Copyright and moral rights for the publications made accessible in the public portal are retained by the authors and/or other copyright owners and it is a condition of accessing publications that users recognise and abide by the legal requirements associated with these rights.

- Users may download and print one copy of any publication from the public portal for the purpose of private study or research.

- You may not further distribute the material or use it for any profit-making activity or commercial gain

- You may freely distribute the URL identifying the publication in the public portal

If you believe that this document breaches copyright please contact us providing details, and we will remove access to the work immediately and investigate your claim. 


\title{
Ultranarrow polaritons in a semiconductor microcavity
}

\author{
J. R. Jensen, ${ }^{\text {a) }}$ P. Borri, ${ }^{\text {b) }}$ W. Langbein, ${ }^{\text {b) }}$ and J. M. Hvam \\ Research Center COM, The Technical University of Denmark, Building 349, DK-2800 Kgs. Lyngby, \\ Denmark
}

(Received 26 January 2000; accepted for publication 4 April 2000)

\begin{abstract}
We have achieved a record high ratio (19) of the Rabi splitting (3.6 meV) to the polariton linewidth $(190 \mu \mathrm{eV})$, in a semiconductor $\lambda$ microcavity with a single $25 \mathrm{~nm}$ GaAs quantum well at the antinode. The narrow polariton lines are obtained with a special cavity design which reduces the exciton broadening due to scattering with free charges and has a very low spatial gradient of the cavity resonance energy. Since the static quantum-well disorder is very small, the polariton broadening is dominantly homogeneous. Still, the measured linewidths close to zero detuning cannot be correctly predicted using the linewidth averaging model. () 2000 American Institute of Physics. [S0003-6951(00)02922-3]
\end{abstract}

Since the first realization of a semiconductor microcavity (MC) structure showing a Rabi splitting of the cavity polaritons $(\mathrm{CP}),{ }^{1}$ the strong-coupling regime of excitons and photons in this two-dimensional system has been studied intensely. Much work has been focused on understanding the behavior of the CP linewidth as a function of detuning, and it is still debated if the linewidths can be fully described using linear dispersion theory or if additional effects such as motional narrowing have to be taken into account. ${ }^{2,3}$ However, little progress has been reported on realizing MCs with $\mathrm{CP}$ lines narrower than $0.5 \mathrm{meV}$, which is interesting, e.g., for bistable devices. ${ }^{4}$

Stanley et al. ${ }^{5}$ have reported a very narrow bare cavity linewidth of $120 \mu \mathrm{eV}$ obtained in a structure grown by molecular-beam epitaxy (MBE). They conclude that the width is limited by the curvature of the thickness of the epitaxial layers across the sample. Bare excitonic resonances of the same width may also be obtained in wide GaAs $/ \mathrm{Al}_{0.3} \mathrm{Ga}_{0.7} \mathrm{As}$ quantum wells at low temperatures, ${ }^{6}$ where the influence of quantum-well disorder is negligible and the homogeneous broadening due to phonon scattering is small. Yet, for a wide GaAs quantum well in a MC, the $\mathrm{CP}$ linewidths that have been obtained are much broader than expected from the bare linewidths. ${ }^{7}$ Instead, the narrowest $\mathrm{CPs}$ have been obtained using a shallow InGaAs/GaAs quantum well where the excitonic resonance is broadened by quantum-well disorder. ${ }^{8}$

In this letter, we demonstrate that ultranarrow $\mathrm{CP}$ lines can be obtained using a single $25 \mathrm{~nm}$ GaAs quantum well confined by a low barrier potential, similar to shallow InGaAs/GaAs quantum wells. Two samples with different barriers, in the following labeled S1 and S2, were grown by MBE for comparison. Both samples consist of a $25 \mathrm{~nm}$ GaAs $/ \mathrm{Al}_{0.3} \mathrm{Ga}_{0.7}$ As quantum well placed at the antinode of a $\lambda$ cavity, with a 25 (16) period $\mathrm{AlAs} / \mathrm{Al}_{0.15} \mathrm{Ga}_{0.85} \mathrm{As}$ Bragg reflector at the bottom (top). In S1 the quantum well is surrounded by $\mathrm{Al}_{0.3} \mathrm{Ga}_{0.7} \mathrm{As}$ barriers forming the spacer layer of

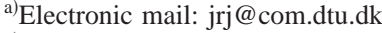

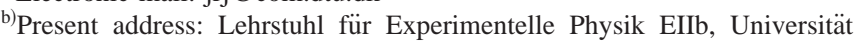
Dortmund, Otto-Hahn Str. 4,D-44221 Dortmund, Germany.
}

the cavity, whereas in S2 the quantum well has 5-nm-wide $\mathrm{Al}_{0.3} \mathrm{Ga}_{0.7} \mathrm{As}$ barriers and the rest of the spacer layer consists of digitally alloyed $\mathrm{Al}_{0.05} \mathrm{Ga}_{0.95}$ As. Both structures were grown with a V/III flux ratio of approximately 8 , a substrate temperature of $630^{\circ} \mathrm{C}$, and a growth rate of $1 \mu \mathrm{m} / \mathrm{h}$. The samples were rotated during growth of the Bragg reflectors and the quantum well but stopped at a specific angle for the growth of the spacer layers on each side of the quantum well, generating a thickness gradient across the sample.

The photoluminescence (PL) spectra of S1 and S2, shown in Fig. 1, were obtained by exciting the quantum wells with a Ti:sapphire laser through the cavity resonance, which was tuned to an energy more than $30 \mathrm{meV}$ above the exciton resonance. The power density of the laser was $0.3-0.9 \mathrm{~W} / \mathrm{cm}^{2}$. Both the heavy-hole exciton $\left(X_{\mathrm{hh}}\right)$ and the light-hole exciton $\left(X_{\mathrm{lh}}\right)$ are visible, as well as a low-energy shoulder on the $X_{\mathrm{hh}}$ peak. This resonance is ascribed to excitons bound to free carriers (trions), in agreement with measurements showing a constant intensity ratio of the lowenergy shoulder to the $X_{\text {hh }}$ peak over two orders of magnitude of the excitation power. The full width at half maximum (FWHM) of the heavy-hole exciton peak in S1 (S2) is $440 \mu \mathrm{eV}(220 \mu \mathrm{eV})$. From the PL spectra we conclude that a significantly larger free-carrier density is present in S1 than in S2, giving rise to a more intense trion peak and
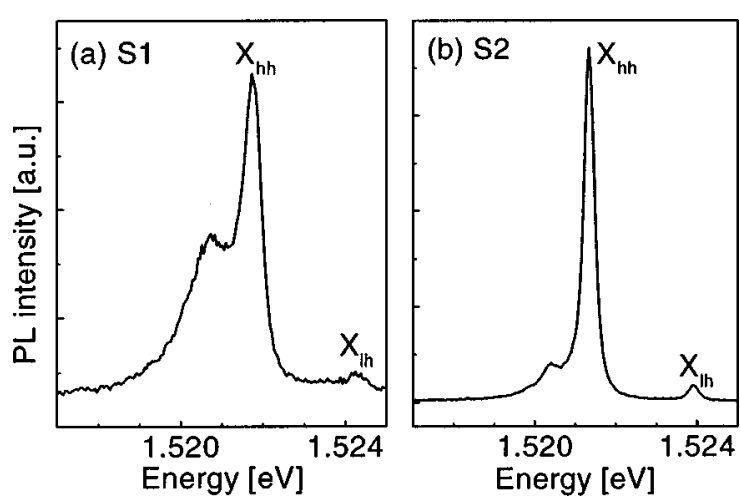

FIG. 1. Photoluminescence spectra of (a) S1 and (b) $\mathrm{S} 2$ at $T=11 \mathrm{~K}$. The labeled peaks correspond to the heavy-hole $\left(X_{\mathrm{hh}}\right)$ and light-hole $\left(X_{\mathrm{lh}}\right)$ excitons. 

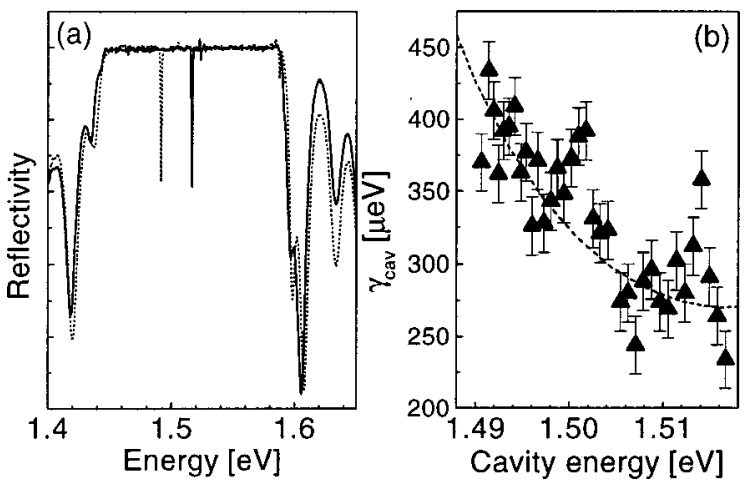

FIG. 2. (a) Reflection spectra of $\mathrm{S} 2(T=11 \mathrm{~K})$ at two different positions on the sample, where the cavity resonance is tuned below the excitonic resonance. (b) The measured (triangles) and calculated (dashed line) Lorentzian cavity linewidths (FWHM) as a function of the cavity resonance energy.

larger homogeneous broadening due to a higher free-carrier scattering rate. A broadening of the excitonic resonance due to charged states has also been reported for other MC structures. ${ }^{9}$

Since S1 and S2 are nominally undoped, the charges in the structures most likely originate from background $p$ doping, due to carbon incorporation during growth. The largest contribution is expected from the AlAs Bragg layers, since previous experiments have shown that the background doping concentration increases with the $\mathrm{Al}$ molefraction in AlGaAs. ${ }^{10}$ Hence, the total concentration of dopants is approximately the same in S1 and S2, but due to the lower band gap of the spacer layer in S2 a smaller density of free charges can accumulate in the quantum well before bandbending makes it energetically favorable to tunnel out into the spacer layer.

In Fig. 2 the broadband reflectivity spectrum is shown for two positions on S2 $18 \mathrm{~mm}$ apart. For all the reflectivity measurements reported here, white light was focused to a diffraction-limited spot size of $75 \mu \mathrm{m}(\mathrm{NA}=0.0065)$ on the sample at normal incidence. For this value of the numerical aperture, the angle of the light cone is small enough to avoid a broadening of the cavity linewidth ${ }^{5}$ due to the $k$ dispersion, and at the same time the spot size is so small that the shift of the cavity energy within the probe spot is always significantly less than the cavity linewidth. The measurements shown in Fig. 2 were obtained for the cavity energy tuned below the exciton resonance, where the properties of the bare cavity can be obtained. As expected, the position of the stop band is the same since the Bragg reflectors have a uniform thickness, but due to the wedge of the spacer layer the cavity resonance energy is shifted. The gradient of the cavity resonance energy is $1.5 \mathrm{meV} / \mathrm{mm}$, which is a factor of 5 lower than what is obtained with our MBE system if the whole MC is wedged. This large reduction of the gradient is in agreement with theoretical calculations showing that the cavity resonance energy is more dependent on the penetration depth into the Bragg reflectors than the spacer layer thickness. ${ }^{11}$

The low gradient of the cavity energy achieved here is very important in order to avoid a broadening of the cavity linewidth due to the shift of the cavity energy within the optimal probe spot area. ${ }^{5}$ However, with this design the width of the cavity resonance is not constant across the structure, as shown in Fig. 2. Since the reflectivity of a Bragg Downloaded 05 Feb 2010 to 192.38.67.112. Redistribution subject
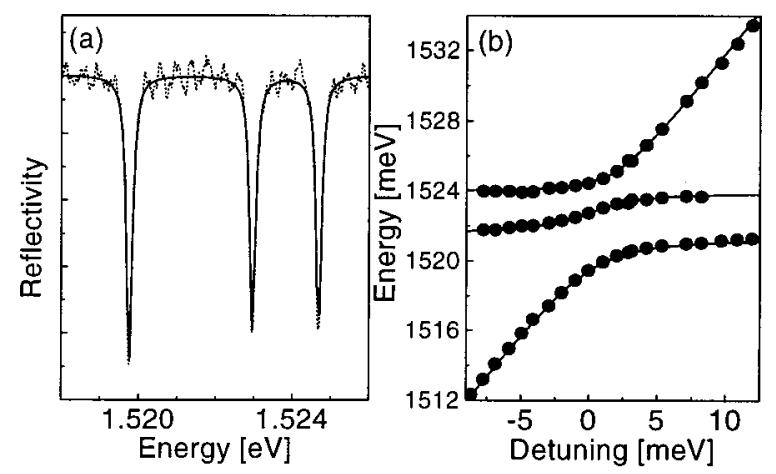

FIG. 3. (a) Measured reflection spectrum (dashed line) of S2 at zero detuning, and best fit obtained with three Lorentzian lines (solid line). (b) Measured (circles) and fitted (solid lines) energies of the polaritons as a function of detuning.

reflector is highest in the middle of the stop band, the cavity linewidth increases when it is detuned from the center due to a reduced photon lifetime in the cavity. The dashed line indicates the calculated cavity linewidths for S2 obtained from a tranfer matrix model. Except for two regions, where the measured linewidths are significantly larger than the calculated values, the agreement is very good. We attribute the increased linewidth to absorption features below the exciton resonance in the quantum well, probably due to defect states. We conclude that within a region of $15 \mathrm{meV}$ from the center of the stop band the measured cavity linewidth is practically constant in such a structure, and theoretically it only increases by $10 \%$.

The reflection spectrum of S2 for the cavity resonance tuned to the energy of the heavy-hole exciton is shown in Fig. 3. The three CP lines correspond to the mixed states between the bare cavity, and the heavy- and light-hole excitons. All three lines are well fitted by Lorentzian line having the same width of $\gamma=190 \mu \mathrm{eV}$, indicating a dominating homogeneous broadening. Indeed, for the $25 \mathrm{~nm}$ quantum well, an inhomogeneous broadening well below $60 \mu \mathrm{eV}$ has been measured, ${ }^{6}$ leaving mostly homogeneous broadening mechanisms for the polaritons, e.g., their radiative decay, and exciton dephasing due to phonon scattering and free-carrier scattering. For these measurements a high-resolution spectrometer was used with a resolution of $30 \mu \mathrm{eV}$. Note that in $\mathrm{S} 1$ the measured reflectivity spectrum showed a much bigger $\mathrm{CP}$ linewidth of $\approx 1 \mathrm{meV}$. The fit of the $\mathrm{CP}$ energies as a function of detuning was obtained by diagonalizing the Hamiltonian of the system,

$$
\left[\begin{array}{ccc}
E_{\mathrm{hh}} & 0 & \hbar \Omega_{\mathrm{hh}} \\
0 & E_{\mathrm{lh}} & \hbar \Omega_{\mathrm{lh}} \\
\hbar \Omega_{\mathrm{hh}} & \hbar \Omega_{\mathrm{lh}} & E_{\mathrm{cav}}
\end{array}\right]\left[\begin{array}{c}
c_{1} \\
c_{2} \\
c_{3}
\end{array}\right]=E\left[\begin{array}{c}
c_{1} \\
c_{2} \\
c_{3}
\end{array}\right],
$$

where $E_{\mathrm{cav}}, E_{\mathrm{hh}}$ and $E_{\mathrm{lh}}$ are the energies of the bare cavity resonance, heavy-hole exciton, and light-hole exciton, and the detuning is $E_{\mathrm{cav}}-E_{\mathrm{hh}} . \hbar \Omega_{\mathrm{hh}}$ and $\hbar \Omega_{\mathrm{lh}}$ are the coupling strengths of the photon to the heavy-hole and light-hole excitons, and correspond to half the Rabi splittings. For the fit, the values of $E_{\mathrm{hh}}$ and $E_{\mathrm{lh}}$ were fixed to the transition energies obtained from the PL measurement, and we found $\hbar \Omega_{\mathrm{hh}}$ $=1.8 \mathrm{meV}$ and $\hbar \Omega_{\mathrm{lh}}=1.1 \mathrm{meV}$, in good agreement with previous measurements. ${ }^{12}$ The ratio of the heavy-hole Rabi to AIP license or copyright; see http://apl.aip.org/apl/copyright.jsp 


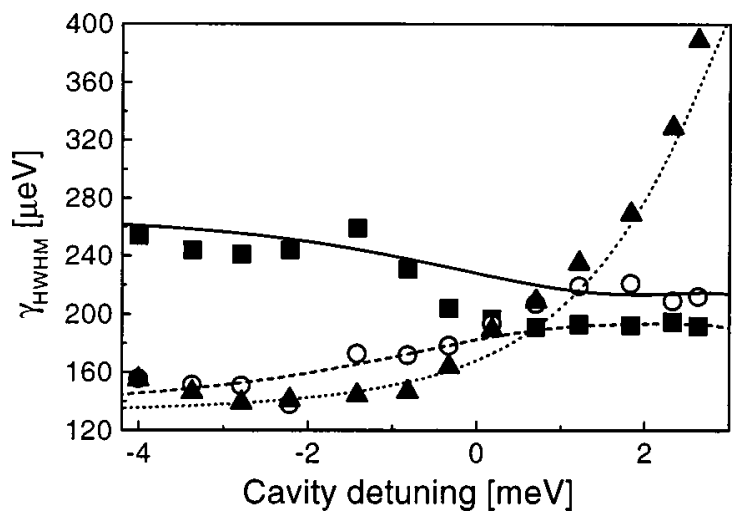

FIG. 4. Comparison of the measured linewidths (FWHM) with the linewidth averaging model [Eq. (2)]. The symbols show the measured values and the lines show the calculated values for the lower polariton (filled squares/solid line), the middle polariton (open circles/dashed line), and the upper polariton (filled triangles/dotted line).

splitting to the linewidth in this sample is $2 \hbar \Omega_{\mathrm{hh}} / \gamma=19$, which is approximately a factor of 2 better than the highest value reported so far. ${ }^{8}$

In Fig. 4, the measured linewidths of the $\mathrm{CP}$ branches are shown as a function of detuning at $T=11 \mathrm{~K}$. Due to the shallow reflectivity dips obtained for the exciton-like polaritons at large detunings, precise fits of all the linewidths could not be obtained over the full detuning range shown in Fig. 3. For negative detuning the mixing of the three bare states is weak, and we observe almost constant linewidths of the CPs. In this region, the width of the lower $\mathrm{CP}$ corresponds to the bare cavity, and the value is in good agreement with the measurements described previously. The widths of the middle and upper CPs correspond to the nonradiative broadening of the heavy- and light-hole excitons, ${ }^{13}$ however, they are $60-80 \mu \mathrm{eV}$ below the values obtained from PL. This difference is attributed to the different experimental conditions of the two measurements, e.g., the exciton resonance observed in the PL may be broadened due to photoinduced charges in the quantum well. Around zero detuning the linewidths cross, as the excitonic and photonic contents of the polaritons change due to the strong mixing. For positive detuning, the upper polariton has a dominating photonic content, so the abrupt increase in the linewidth observed here is explained by increased absorption in the cavity resonance due to the proximity of the continuum edge of the quantum well.

In order to compare the measurements with theory, we have calculated the CP linewidths using the linewidth averaging model,

$$
\gamma_{\mathrm{pol}}=\left|c_{1}\right|^{2} \gamma_{\mathrm{hh}}+\left|c_{2}\right|^{2} \gamma_{\mathrm{lh}}+\left|c_{3}\right|^{2} \gamma_{\mathrm{cav}}
$$

Here, the nonradiative linewidths of the excitonic states were assumed to be constant, $\gamma_{\mathrm{hh}}=\gamma_{\mathrm{lh}}=130 \mu \mathrm{eV}$. The change of $\gamma_{\text {cav }}$ due to absorption was empirically modeled using the tail of a Lorentzian, and assuming a constant 270 $\mu \mathrm{eV}$ linewidth in the absence of absorption.

We note two important differences between the measured and calculated values. First, the measured lower CP linewidth drops more steeply close to zero detuning than the calculated value, and second, the crossing of the upper and lower CP linewidths is shifted about $1 \mathrm{meV}$ below the calculated crossing point. Both the steepness and the crossing Downloaded 05 Feb 2010 to 192.38.67.112. Redistribution subject point are practically insensitive to variations in $\gamma_{\mathrm{hh}}, \gamma_{\mathrm{lh}}$, and $\gamma_{\text {cav }}$, and almost entirely determined by the values of $c_{1}, c_{2}$, and $c_{3}$. However, the accurate fit of the eigenvalues in Fig. 3 show that $c_{1}, c_{2}$, and $c_{3}$ are well determined. We emphasize that our structure is a three-level system while most other structures studied both experimentally and theoretically are two-level systems, which implies that zero detuning in our definition $\left(E_{\mathrm{hh}}=E_{\mathrm{cav}}\right)$ is not directly comparable to zero detuning in other structures. It is more relevant to compare with the detuning for which the upper and lower polaritons have equal excitonic and photonic contents, which is at $1.2 \mathrm{meV}$ in S2. Indeed, at this value of the detuning, the lower CP is narrower than the upper $\mathrm{CP}$, as predicted in Ref. 14. Temperature-dependent measurements have been carried out in order to investigate this effect further, which are described elsewhere. ${ }^{15}$

In conclusion, we have shown that MCs with ultranarrow homogeneously broadened polariton lines can be realized with a GaAs quantum well if a cavity design with shallow energy levels is used, reducing the density of free carriers in the well. Wedging only the spacer layer during growth ensures a low gradient of the cavity energy, and we have experimentally verified that for such a structure the variation of the cavity linewidth is negligible in a range of 15 $\mathrm{meV}$ from the center of the stop band, in agreement with transfer matrix calculations. Finally, we observe that close to zero detuning the polariton linewidths are not well predicted by the linewidth averaging model, indicating that additional narrowing of the lower polariton is taking place.

The authors would like to thank K. Leosson and N. A. Mortensen for help on the high-resolution measurements and parts of the numerical calculations. Also, the fruitful discussions with C. B. Sørensen at the III-V Nanolab on the MBE growth are acknowledged. This work was supported by the Danish Natural Science Research Council.

${ }^{1}$ C. Weisbuch, M. Nishioka, A. Ishikawa, and Y. Arakawa, Phys. Rev. Lett. 69, 3314 (1992)

${ }^{2}$ C. Ell, J. Prineas, Jr., S. Park, H. M. Gibbs, G. Khitrova, S. W. Koch, and R. Houdre, Phys. Rev. Lett. 80, 4795 (1998).

${ }^{3}$ D. M. Whittaker, P. Kinsler, T. A. Fisher, M. S. Skolnick, A. Armitage, A. M. Afshar, M. D. Sturge, and J. S. Roberts, Phys. Rev. Lett. 77, 4792 (1996).

${ }^{4}$ D. Citrin and T. B. Norris, IEEE J. Sel. Top. Quantum Electron. 2, 401 (1996).

${ }^{5}$ R. P. Stanley, R. Houdre, U. Oesterle, M. Gailhanou, and M. Ilegems, Appl. Phys. Lett. 65, 1883 (1994).

${ }^{6}$ W. Langbein and J. M. Hvam, Phys. Rev. B 61, 1692 (2000).

${ }^{7}$ S. Pau, G. Björk, H. Cao, F. Tassone, R. Huang, and Y. Yamamoto, Phys. Rev. B 55, R1942 (1997).

${ }^{8}$ J. D. Berger, O. Lyngnes, H. M. Gibbs, G. Khitrova, T. R. Nelson, E. K. Lindmark, A. V. Kavokin, M. A. Kaliteevski, and V. V. Zapasskii, Phys. Rev. B 54, 1975 (1996).

${ }^{9}$ R. P. Stanley, R. Houdre, U. Oesterle, and M. Ilegems, Solid State Commun. 106, 485 (1998).

${ }^{10}$ C. B. Sørensen (private communications).

${ }^{11}$ G. Panzarini, L. C. Andreani, A. Armitage, D. Baxter, M. S. Skolnick, V. N. Astratov, J. S. Roberts, A. V. Kavokin, M. R. Vladimirova, and M. Kaliteevski, Phys. Rev. B 59, 5082 (1999).

${ }^{12}$ H. Cao, S. Jiang, S. Machida, Y. Takiguchi, and Y. Yamamoto, Appl. Phys. Lett. 71, 1461 (1997)

${ }^{13}$ V. Savona, L. C. Andreani, P. Schwendimann, and A. Quattropani, Solid State Commun. 93, 733 (1995).

${ }^{14}$ V. Savona and C. Piermarocchi, Phys. Status Solidi A 164, 45 (1997).

${ }^{15}$ P. Borri, J. R. Jensen, W. Langbein, and J. M. Hvam, Phys. Rev. B (to be published).

AIP license or copyright; see http://apl.aip.org/apl/copyright.jsp 